\title{
Accuracy analysis of GPS/BDS relative positioning using zero-baseline measurements
}

\author{
Gethin Wyn Roberts ${ }^{1 *}, \mathrm{Xu} \mathrm{Tang}^{2}$ and Xiufeng $\mathrm{He}^{3}$
}

\begin{abstract}
This paper focuses on assessing the precision of carrier phase relative positioning using GPS-only, BDS-only and GPS/BDS measurements. A zero baseline is used in order to achieve this. Software for GPS and BDS processing has been developed, allowing static and kinematic data processing, as well as the combined GPS and BDS processing. lonospheric and tropospheric delays are significantly reduced by double differencing between satellites and receivers, but the Multipath signals are still a major source of error for the various general GNSS baseline applications. In this paper, two Multi-GNSS receivers are connected to one antenna by an antenna splitter. This strategy results in all the delays or errors being mitigated, leaving only the random measurement noises resulted from the double difference processing. The time series of the final baseline error reveal that both GPS and BDS can achieve a precision of millimetres, but GPS performs better than BDS. Results from the combined processing of GPS and BDS demonstrate that the integration of GPS and BDS can significantly improve the precision, compared with the GPS-only and BDS-only results.
\end{abstract}

Keywords: BDS satellite navigation system, Integration of GPS and BDS, Kinematic positioning error

\section{Introduction}

China is developing its own version of GPS, called BDS (BeiDou satellite navigation System). Currently, BDS has 14 satellites distributed in three different kinds of orbits, as seen in Table 1. The five satellites known as $\mathrm{C} 1, \mathrm{C} 2, \mathrm{C} 3, \mathrm{C} 4, \mathrm{C} 5$ are located in Geostationary Earth Orbits (GEO); C6 to C10 are located in Inclined Geo-Synchronous Orbits (IGSO); the remainder are located in Medium Earth Orbits (MEO). The BDS MEO's orbital altitude is around 21,500 km, a similar height to GPS, GLONASS and Galileo satellite navigation systems. Currently, BDS is operational mainly over China and the surrounding area, with a plan for BDS to be fully international by 2020 . Both the GEO and IGSO satellites are located over China, but the BDS MEO satellites can also be tracked by users from non-Asia-Pacific regions. Figure 1 illustrates the distribution of the 14 BDS satellites. The satellites' tracks in Fig. 1 were calculated using an ephemeris from the 27th December 2012. There will be more MEO satellites launched between 2015 and 2020, which is the second stage

\footnotetext{
* Correspondence: Gethinr@setur.fo

${ }^{1}$ Faculty of Natural Sciences and Technology, University of the Faroe Islands, Tórshavn, Faroe Islands

Full list of author information is available at the end of the article
}

of the BDS project. The planned future global BDS constellation will consist of 5 GEO, three IGSO and 27 MEO satellites by 2020 (BeiDou-SIS-ICD-Test 2011).

The first test satellite, called M1, was launched in 2007. The BDS code structure is analyzed by using high gain parabolic antennas at two stations located at (Grelier et al. 2007) CNES (Toulouse, France) and Leeheim (Germany). Gao et al. decoded the M1 satellite's codes in the E2, E5b and E6 bands, extracted the code bits, and derived the code generators (Gao et al. 2009). All GPS satellites' ephemerides can employ a uniform parameter, as the GPS constellation consisted of only MEO satellites. The BDS constellation is a mixture of GEO, IGSO and MEO satellites. A method has been presented to design optimal parameter sets for all these three types of satellites (Fu and Wu 2011). The clock offset was estimated by processing the BDS M1 observations based on the orbit solution from laser ranging measurements (Hauschild et al. 2012). Unexpectedly high dynamics in the clock results were found, which affect both the pseudo-range and carrier phase measurements.

Since the increase of BDS satellite numbers, many researches have focused on assessing BDS position precision, orbital determination and integration of BDS with other 
Table 1 Current BDS Operational Satellites (October 2014)

\begin{tabular}{llllll}
\hline PRN & Type & Launch Time & Longitude & Latitude & Approximate Height \\
\hline C01 & GEO & $2010 / 01 / 07$ & $140.07^{\circ}$ & $\sim 0^{\circ}$ & $\sim 36,000 \mathrm{~km}$ \\
C02 & GEO & $2012 / 10 / 25$ & $80.22^{\circ}$ & $\sim 0^{\circ}$ & $\sim 36,000 \mathrm{~km}$ \\
C03 & GEO & $2010 / 06 / 02$ & $110.56^{\circ}$ & $\sim 0^{\circ}$ & $\sim 36,000 \mathrm{~km}$ \\
C04 & GEO & $2010 / 11 / 01$ & $160.00^{\circ}$ & $\sim 0^{\circ}$ & $\sim 36,000 \mathrm{~km}$ \\
C05 & GEO & $2012 / 02 / 25$ & $58.65^{\circ}$ & $\sim 0^{\circ}$ & $\sim 36,000 \mathrm{~km}$ \\
C06 & IGSO & $2010 / 08 / 01$ & $104.63^{\circ} \mathrm{E}-136.05^{\circ} \mathrm{E}$ & $54: 61^{\circ} \mathrm{S}-54: 61^{\circ} \mathrm{N}$ & $\sim 36,000 \mathrm{~km}$ \\
C07 & IGSO & $2010 / 12 / 18$ & $102.60^{\circ} \mathrm{E}-134.13^{\circ} \mathrm{E}$ & $54: 81^{\circ} \mathrm{S}-54: 81^{\circ} \mathrm{N}$ & $\sim 36,000 \mathrm{~km}$ \\
C08 & IGSO & $2011 / 04 / 10$ & $100.49^{\circ} \mathrm{E}-133.82^{\circ} \mathrm{E}$ & $56: 02^{\circ} \mathrm{S}-56: 02^{\circ} \mathrm{N}$ & $\sim 36,000 \mathrm{~km}$ \\
C09 & IGSO & $2011 / 07 / 27$ & $80.12^{\circ} \mathrm{E}-111.79^{\circ} \mathrm{E}$ & $54: 93^{\circ} \mathrm{S}-54: 93^{\circ} \mathrm{N}$ & $\sim 36,000 \mathrm{~km}$ \\
C10 & IGSO & $2011 / 12 / 02$ & $78.66^{\circ} \mathrm{E}-110.33^{\circ} \mathrm{E}$ & $54: 93^{\circ} \mathrm{S}-54: 93^{\circ} \mathrm{N}$ & $\sim 36,000 \mathrm{~km}$ \\
C11 & MEO & $2012 / 04 / 30$ & $180^{\circ} \mathrm{E}-180^{\circ} \mathrm{W}$ & $55: 31^{\circ} \mathrm{S}-54: 61^{\circ} \mathrm{N}$ & $\sim 21,500 \mathrm{~km}$ \\
C12 & MEO & $2012 / 04 / 30$ & $180^{\circ} \mathrm{E}-180^{\circ} \mathrm{W}$ & $55: 25^{\circ} \mathrm{S}-54: 81^{\circ} \mathrm{N}$ & $\sim 21,500 \mathrm{~km}$ \\
C13 & MEO & $2012 / 09 / 19$ & $180^{\circ} \mathrm{E}-180^{\circ} \mathrm{W}$ & $54: 99^{\circ} \mathrm{S}-56: 02^{\circ} \mathrm{N}$ & $\sim 21,500 \mathrm{~km}$ \\
C14 & MEO & $2012 / 09 / 19$ & $180^{\circ} \mathrm{E}-180^{\circ} \mathrm{W}$ & $55: 10^{\circ} \mathrm{S}-59: 93^{\circ} \mathrm{N}$ & $\sim 21,500 \mathrm{~km}$ \\
\hline
\end{tabular}

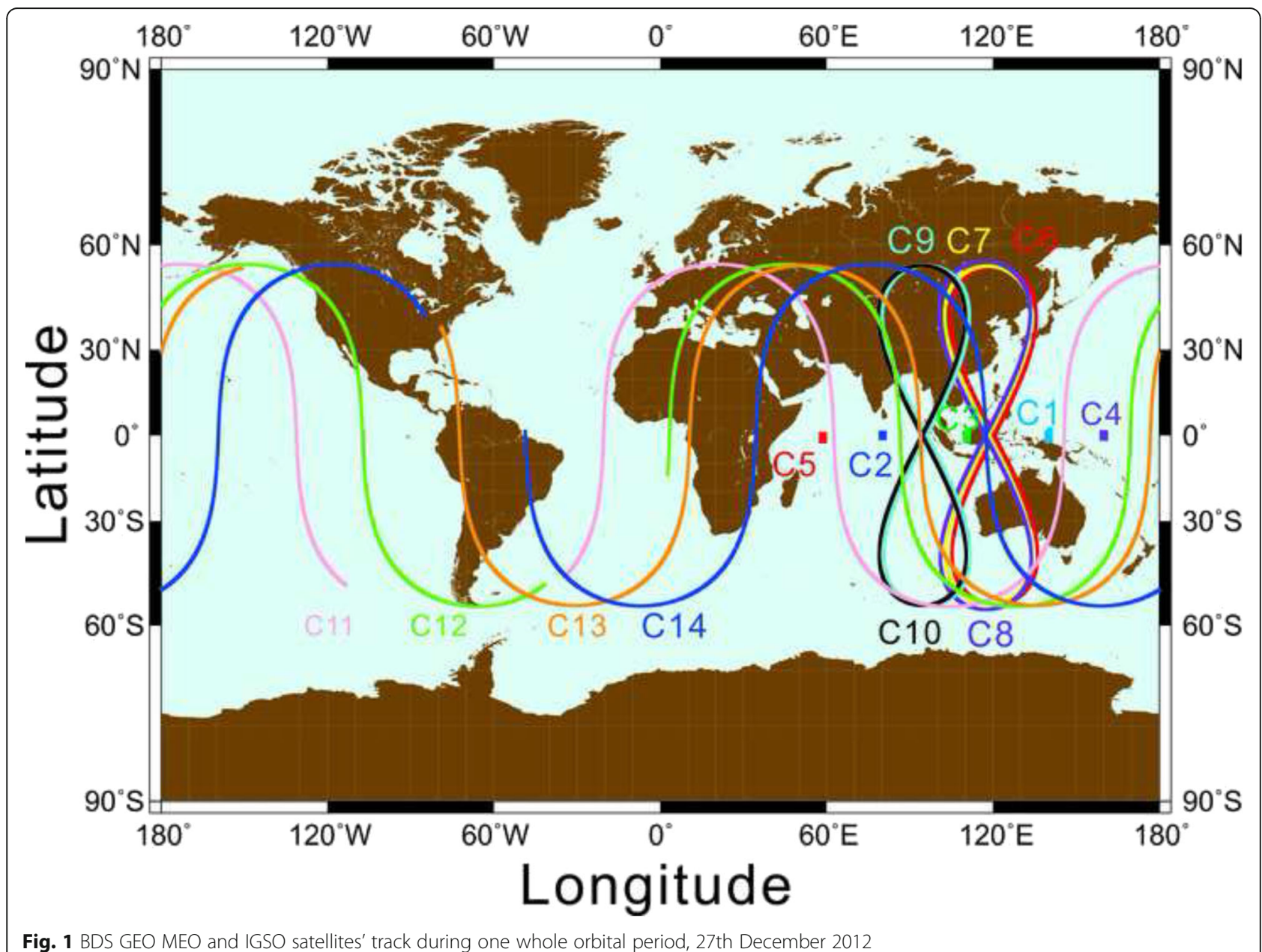

Fig. 1 BDS GEO MEO and IGSO satellites' track during one whole orbital period, 27th December 2012 
satellite navigation systems. (Shi et al. 2012) carried out early research, using one week of BDS GEO and IGSO satellites' observations to assess the preliminary positioning performance. Their work revealed that BDS code measurements' noise was higher than GPS's. Both data were collected using the same GPS/BDS receiver. The precise relative positioning using code and carrier phase measurements revealed that BDS precision is better than $2 \mathrm{~cm}$ in the North-South component and $4 \mathrm{~cm}$ at the vertical component. The standard deviation of the East-West component is smaller than $1 \mathrm{~cm}$. The BDS code measurements using 5 GEO, 5 IGSO and 4 MEO satellites' carrier phase measurements were assessed in both static and kinematic positioning (Tang et al. 2014; Tang 2014). Researchers have combined BDS with other satellite navigation systems in order to assess the short baseline ambiguity resolution reliability, and the availability in high cut-off elevation situation (He et al. 2014; Deng et al. 2013; Odolinski et al. 2014; Teunissen et al. 2014). Multipath delay is one of the main error sources, which is a limitation of GNSS positioning accuracies improving (Wang et al. 2014). The BDS GEO satellites' multipath are even more difficult to mitigate due to the relatively stationary geometry when compared to MEO satellites in particular.

This paper assesses the precision of GPS and BDS carrier phase positioning using the current constellations. The results of this study reveal that by integrating both GPS and $\mathrm{BDS}$, it is possible to improve the precision compared to GPS-only and BDS-only results. This paper is organized as follows. In section 2, the zero-baseline experiment is briefly described. In section 3, the GPS and BDS satellites' availability is detailed. In section 4, the GPS-only, BDS-only and combined GPS and BDS kinematic results are presented.

\section{Results and discussion}

\section{Zero baseline data collection} Field experiments

Two ComNav K508 receivers were connected to one antenna using a GEMS signal splitter (PN:GS18). These two receivers have the capacity of tracking GPS L1, L2 and L5 signals, BDS B1, B2 and B3 signals, as well as the two GLONASS signals. As these two receivers tracked the satellite signals at the same antenna phase center, observations from the two receivers could be assumed as a baseline having a distance of "zero" units. The time series results of the final baseline processing reveal the precision of the two satellite navigation systems, as compared to the known distance of zero units. $24 \mathrm{~h}$ (one whole BDS orbital period, UTC 00:00:00-23:59:59) of data were collected on April 7th 2014. A Leica AR 25 choke ring antenna was located on a pillar on the roof of the Faculty of Science and Engineering building, University of Nottingham Ningbo China (UNNC) (Fig. 2). The cable connecting the Leica AR25 choke ring antenna and the signal splitter is around $50 \mathrm{~m}$ long, and two $3 \mathrm{~m}$ long antenna cables were used to connect the splitter with the two ComNav receivers. Both receivers' sampling rate and the cut-off angle were set as $1 \mathrm{~Hz}$ and $15^{\circ}$, respectively. The zero-baseline data were processed using the software developed at UNNC.

\section{Zero baseline error analysis}

Considering the two receivers' site names as $i$ and $j$, and the two tracked satellites' names as $p$ and $q$. Considering the tropospheric delay, ionospheric delay, multipath error, satellite and receiver clock errors, as well as the random receiver noise errors, these two receiver observation equations to satellite $p$ can be written as:

$$
\begin{aligned}
\phi_{i}^{p}= & \rho_{i}^{p}+c\left(\delta t^{p}-\delta t_{i}\right)+N_{i}^{p}+T_{i}^{p}+I_{i}^{p}+M_{i}^{p} \\
& +\xi_{i}^{p} \\
\phi_{j}^{p}= & \rho_{j}^{p}+c\left(\delta t^{p}-\delta t_{j}\right)+N_{j}^{p}+T_{j}^{p}+I_{j}^{p}+M_{j}^{p}+\xi_{j}^{p}
\end{aligned}
$$

where $\phi$ is the carrier phase measurement; $\rho$ is the geometrical range between satellite $p$ and receivers $i$ and $j ; c$ is the speed of light in a vacuum. $\delta t^{p}$ is the satellite $p$ clock error; $\delta t_{i}$ and $\delta t_{j}$ are the receiver $i$ and receiver $j$ clock errors, respectively; $N_{i}^{p}$ and $N_{j}^{p}$ are the integer ambiguities of receivers $i$ and $j$ from satellite $p$ carrier phase measurements, respectively. $T_{i}^{p}$ and $T_{j}^{p}$ are the tropospheric delays of receivers $i$ and $j$ from satellite $p$, respectively; $I_{i}^{p}$ and $I_{j}^{p}$ are the ionospheric delays from satellite $p$ to receivers $i$ and $j$, respectively; $M_{i}^{p}$ and $M_{j}^{p}$ are the multipath noise of receivers $i$ and $j$ from satellite $p$. Receivers $i$ and $j$ have the same tropospheric and ionospheric delay when they track the same satellite over a zero baseline. Choke ring antennas have the capacity of resisting most of the multipath. The remaining multipath impacts equally onto receivers $i$ and $j$ in this zero-baseline scenario. The single difference equation between the two receivers can be written as:

$$
\phi_{i, j}^{p}=\rho_{i, j}^{p}-c \delta t_{i, j}+N_{i, j}^{p}+\xi_{i, j}^{p}
$$

Considering another satellite $q$, a second single difference equation can be written as:

$$
\phi_{i, j}^{q}=\rho_{i, j}^{q}-c \delta t_{i, j}+N_{i, j}^{q}+\xi_{i, j}^{q}
$$

where the general relationship $g_{i, j}=g_{j}-g_{i}$. The tropospheric, ionospheric and multipath errors could be totally eliminated by differencing between the receivers' carrier phase data. The random errors which reflect the quality of the signals, single difference integer ambiguity and the single difference receivers' clock error remain. Through calculating the difference between eq. 4 minus eq. 3 , the double difference observation is derived as:

$$
\phi_{i, j}^{p, q}=\rho_{i, j}^{p, q}+N_{i, j}^{p, q}+\xi_{i, j}^{p, q}
$$




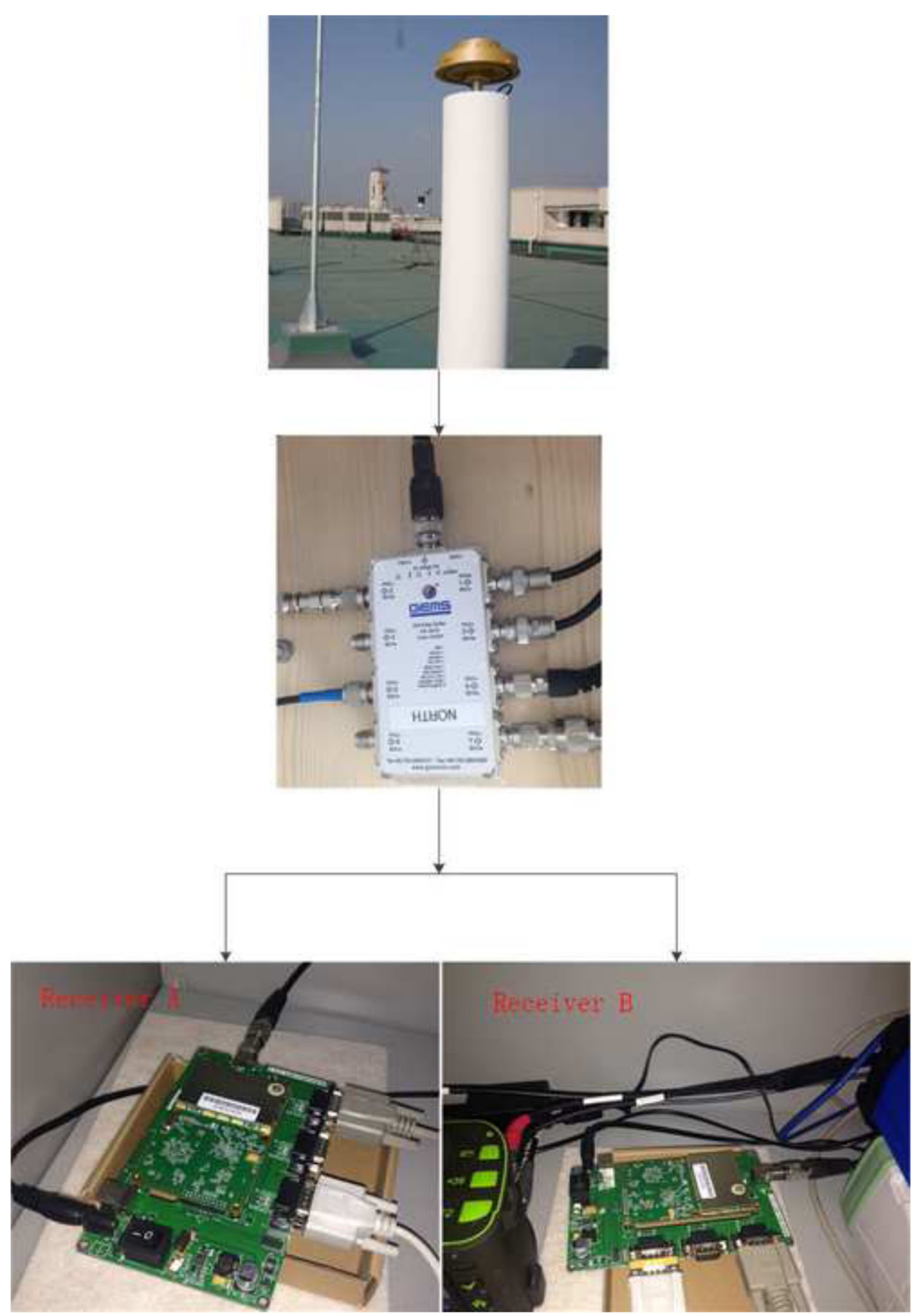

Fig. 2 Zero baseline field experiment; a Leica AR25 choke ring antenna, connected to the two ComNav K508 GNSS receivers via the GEMS signal splitter

where the general relationship for all the individual components is $g_{i, j}^{p, q} \cdot g_{i, j}^{p, q}$ represents the double difference operator, $g_{i, j}^{p, q}=g_{j}^{q}-g_{j}^{p}-g_{i}^{q}+g_{i}^{p}$.

Baseline resolution is also based on the satellite position. The satellites' position derived by the ephemeris include errors, which can reduce the precision of the baseline resolution. The relationship of baseline precision and satellite orbital precision is:

$$
\delta b=\frac{\delta s}{\rho} \cdot b
$$

where $\delta b$ is the error of the baseline, $\delta s$ is the satellite orbital error, $\rho$ is the distance between the tracked satellite to the receiver $b$ is the length of the baseline. In the zero-baseline scenario, the error of the satellite orbit can be eliminated through differencing (Eq. 6). 


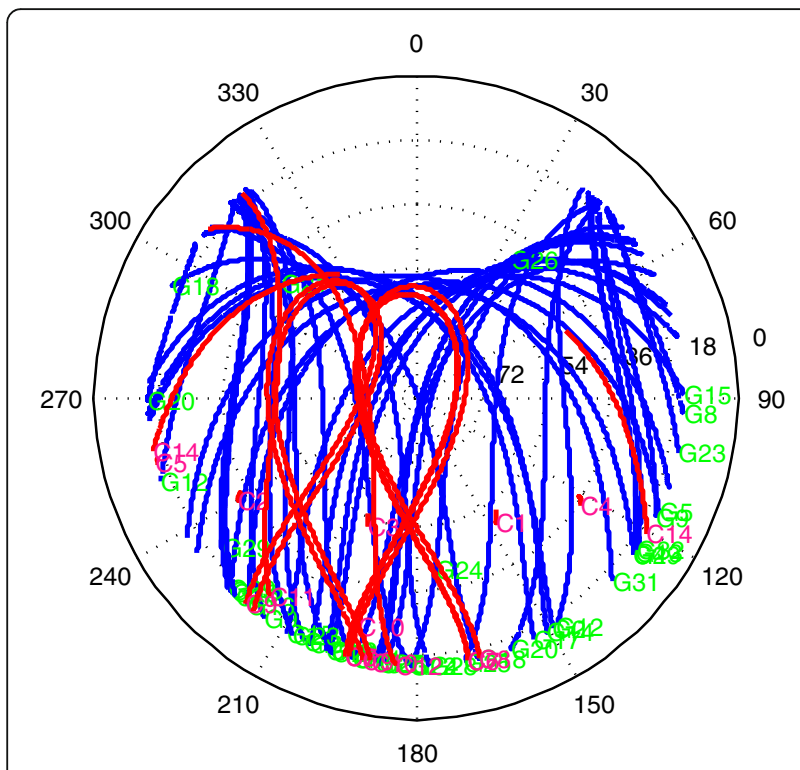

Fig. 3 GPS + BDS sky plot in one whole BDS orbital period (24 h)

Linearizing $\rho_{i, j}^{p, q}$ and rewriting eq. 5 results in:

$$
\phi_{i, j}^{p, q}=\left[\begin{array}{lll}
l_{i, j}^{p, q} & m_{i, j}^{p, q} & n_{i, j}^{p, q}
\end{array}\right] \cdot\left[\begin{array}{lll}
\delta x & \delta y & \delta z
\end{array}\right]^{T}+N_{i, j}^{p, q}+\xi_{i, j}^{p, q}
$$

$\left[l_{i, j}^{p, q} \quad m_{i, j}^{p, q} n_{i, j}^{p, q}\right]$ is the double difference distance from the satellites to the receivers, which could be calculated by the receivers' approximate coordinates and the tracked satellites' coordinates. [ $\delta x \quad \delta y \quad \delta z]$ is the correction of the baseline's 3D distance, $N_{i, j}^{p, q}$ is the double difference integer ambiguity, $\xi_{i, j}^{p, q}$ is the double difference random noise. The precision of the baseline correction is not only determined by the observation noise, but also the coefficient $\left[\begin{array}{lll}l_{i, j}^{p, q} & m_{i, j}^{p, q} & n_{i, j}^{p, q}\end{array}\right]$, after the double difference

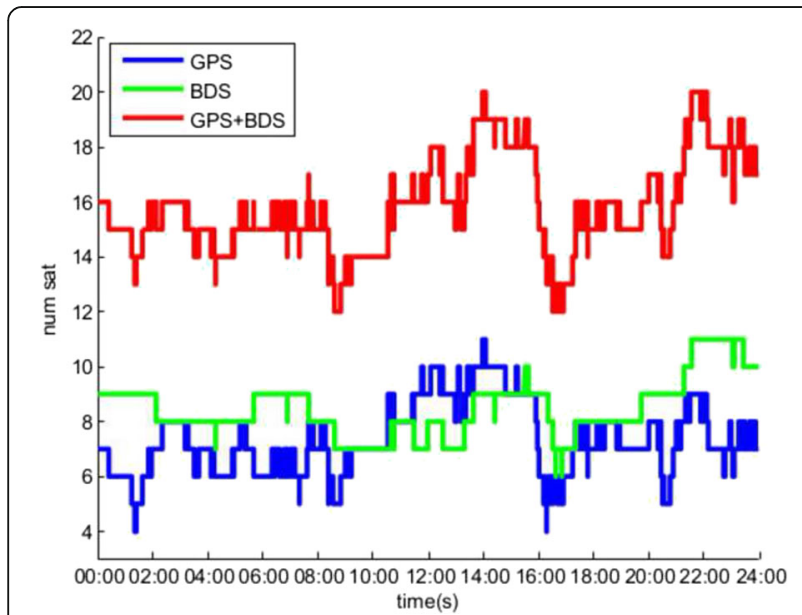

Fig. 4 The number of visible satellites at each epoch over a $24 \mathrm{~h}$

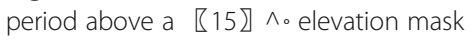

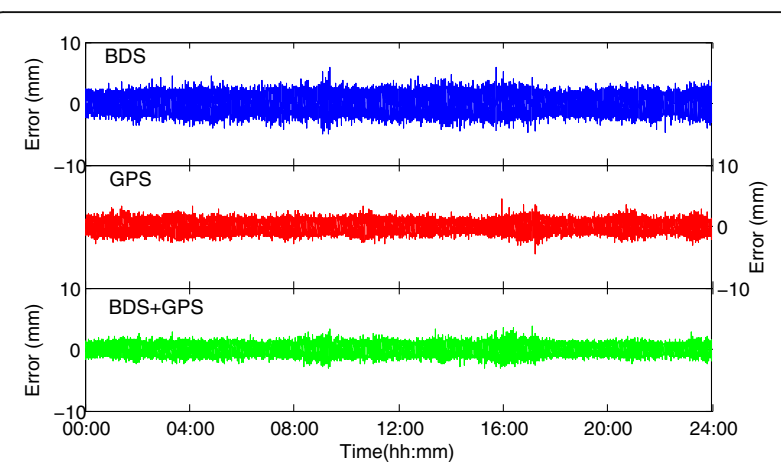

Fig. 5 Position Error in the East-West component for GPS-only, BDSonly and Integration of GPS and BDS

integer ambiguity $N_{i, j}^{p, q}$ is correctly fixed. We use variance of unit weight during the least squares adjustment. The coefficients reflect the efficiency of different satellite geometry. In this paper, the final positional error is a combination of the observation random noise and the satellites' geometrical impact. Distinguishing between these error sources is the topic of ongoing work. This paper pays more attention to the difference of GPS-only and BDS-only position precision and the improvement through the integration of GPS and BDS data.

\section{Satellites' visibility}

In this section, GPS and BDS constellations are analyzed through the whole BDS orbital period observation and ephemeris data. Figure 3 gives the sky plot of GPS (blue) and BDS (red). All the GEO satellites are distributed to the south of the users located in the northern hemisphere (eg Ningbo, China at a location of $29^{\circ} 52^{\prime} N$ and $121^{\circ} 33^{\prime} E$ ). IGSO satellites can be tracked for the most part of their orbital period by the station, but they have the same feature as with MEO satellites in that IGSO satellites can drop out of sight due to their orbit. There is a specific area in the sky where the satellites are never present. This

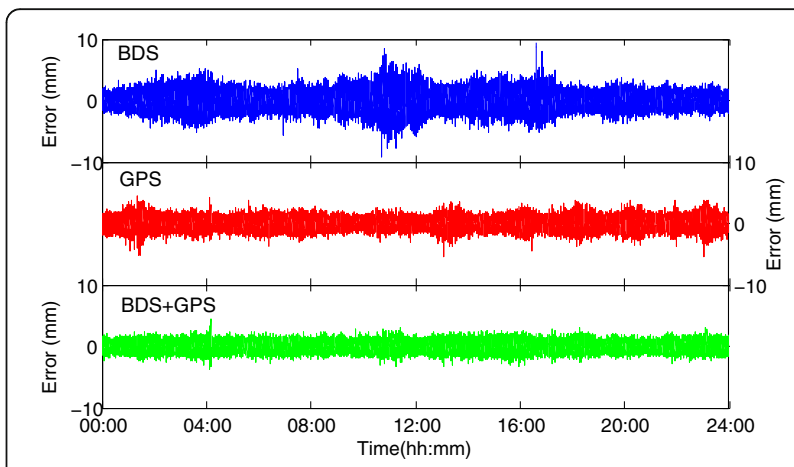

Fig. 6 Position Error in the North-South component for GPS-only, BDS-only and Integration of GPS and BDS 


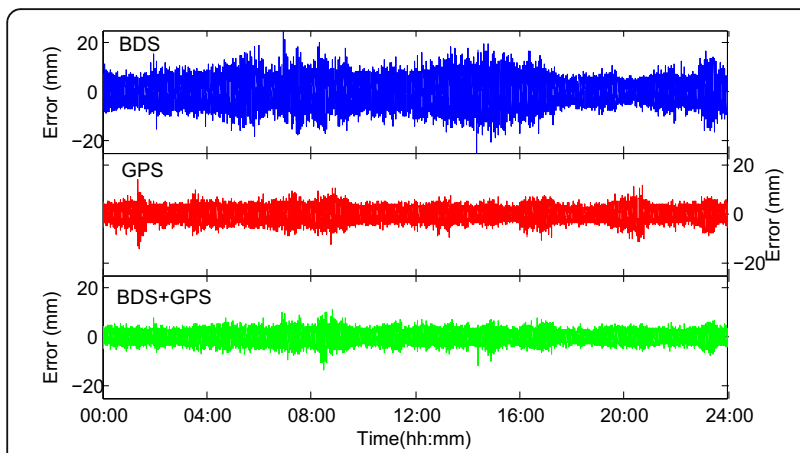

Fig. 7 Position Error in the Height component for GPS-only, BDSonly and Integration of GPS and BDS

area is located in the north of the sky plot to the northern hemisphere users.

Figure 4 illustrates the number of GPS and BDS satellites visible over an elevation mask of $15^{\circ}$. It demonstrates that there are only 4 GPS satellites in sight during some epochs, but BDS has at least 6 satellites that could be tracked during the $24 \mathrm{~h}$ period. In some instances, there are more BDS satellites than GPS being tracked. There are at least 12 navigation satellites being tracked when GPS and BDS are combined for positioning. The integration of GPS and BDS can be very useful when the user is located in a non-GNSS friendly environment.

\section{GPS-only, BDS-only and GPS + BDS kinematic results}

The precise carrier phase measurements are gathered by the two receivers connected through the splitter to the single Leica AR 25 choke ring antenna. The antenna is connected to the receivers via a $50 \mathrm{~m}$ long coaxial antenna cable, with

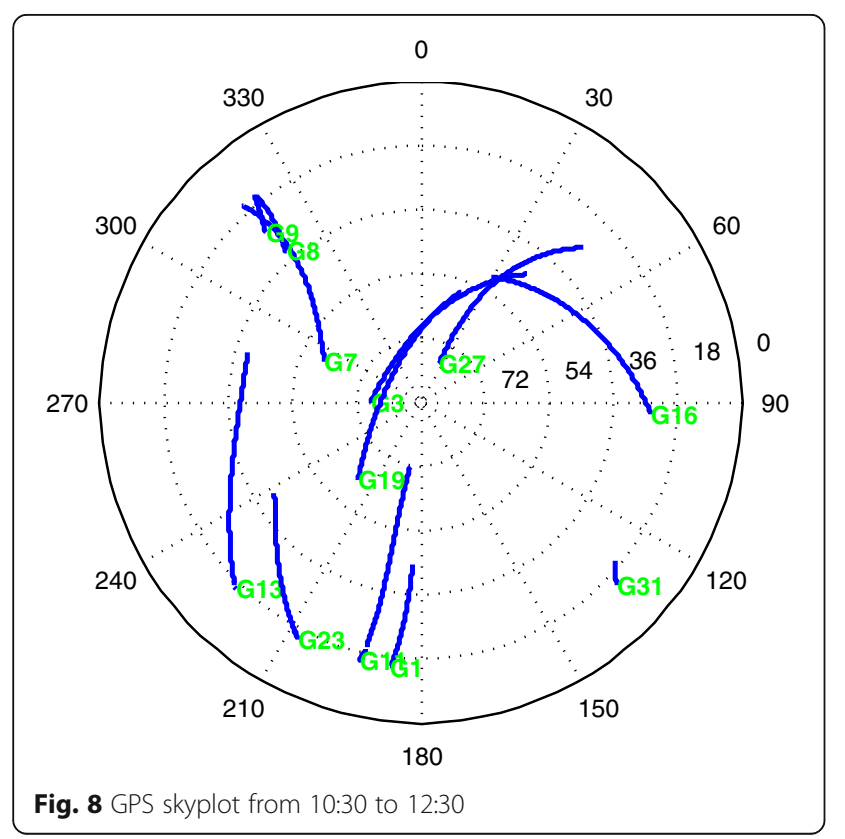

the splitter located at the GNSS receiver end. Tropospheric and ionospheric delays on the two receivers are completely removed by double differencing between the two receivers, as well as the multipath impact. A method for cycle-slip detection was also used for the data pre-process (Liu 2010). The GPS and BDS RINEX data gathered on the 7th of April 2014 were processed epoch by epoch, respectively. Figures 5, 6 and 7 illustrate the time series of positioning errors in the east-west, north-south and height components for GPS, BDS and a combined GPS + BDS solution. GPS position error is no more than $\pm 2 \mathrm{~mm}$ most of the time in both the NorthSouth and the East-West components. BDS position error is no more than $\pm 3 \mathrm{~mm}$ in the east-west component. The BDS precision in the north-south and height components is not as stable as GPS. Errors during 02:00-04:00, 10:00-13:00 and some epoches around 17:00 are observed as being bigger than other times in the north-south component, this also happens in the height component in the BDS positional error time series. Comparing the GPS-only and BDS-only resolutions in Figs. 5, 6 and 7, the GPS positional precision currently performs better than BDS in the three components. This is partly due to the fact that the BDS constellation is still incomplete. Figure 6 reveals that BDS position noise is much more obvious than GPS during 10:30 to 12:30. The GPSonly and BDS-only sky plots, Figs. 8 and 9 respectively, reveal that most of the BDS satellites are located to the south of the station. The GPS satellites' orbital tracks are longer than BDS GEO and IGSO satellites during the $2 \mathrm{~h}$ period. The GPS satellites coverage are also more spread out than BDS during this $2 \mathrm{~h}$ period. The overall conclusion is that the incomplete BDS constellation has a poorer geometrical spread than GPS. However, the integrated GPS/BDS solution has the best spread of satellites. Both GPS and BDS have the same feature

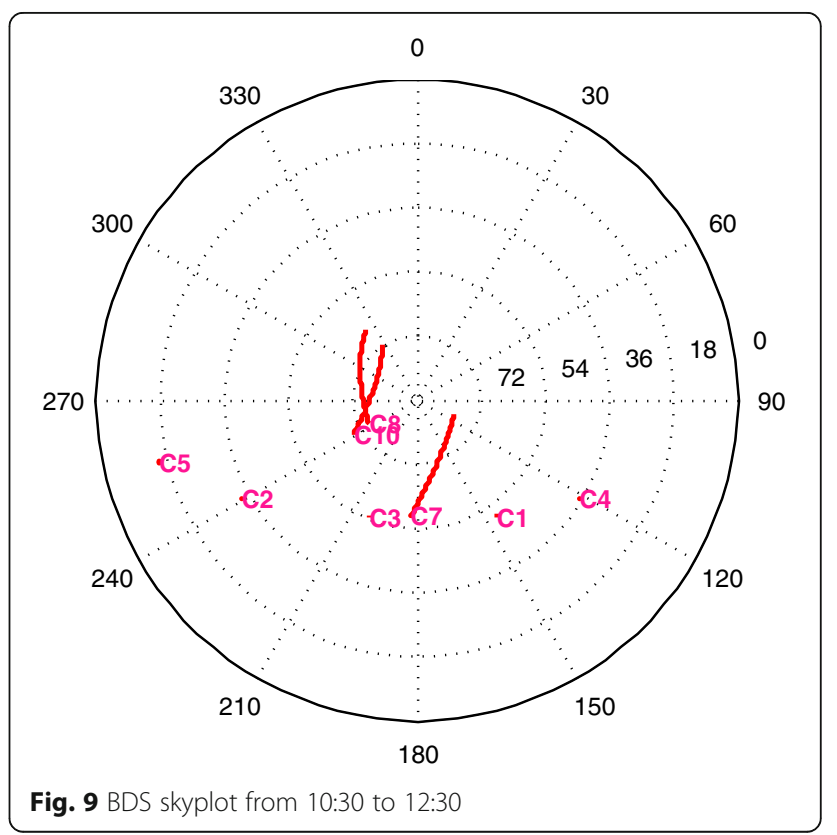



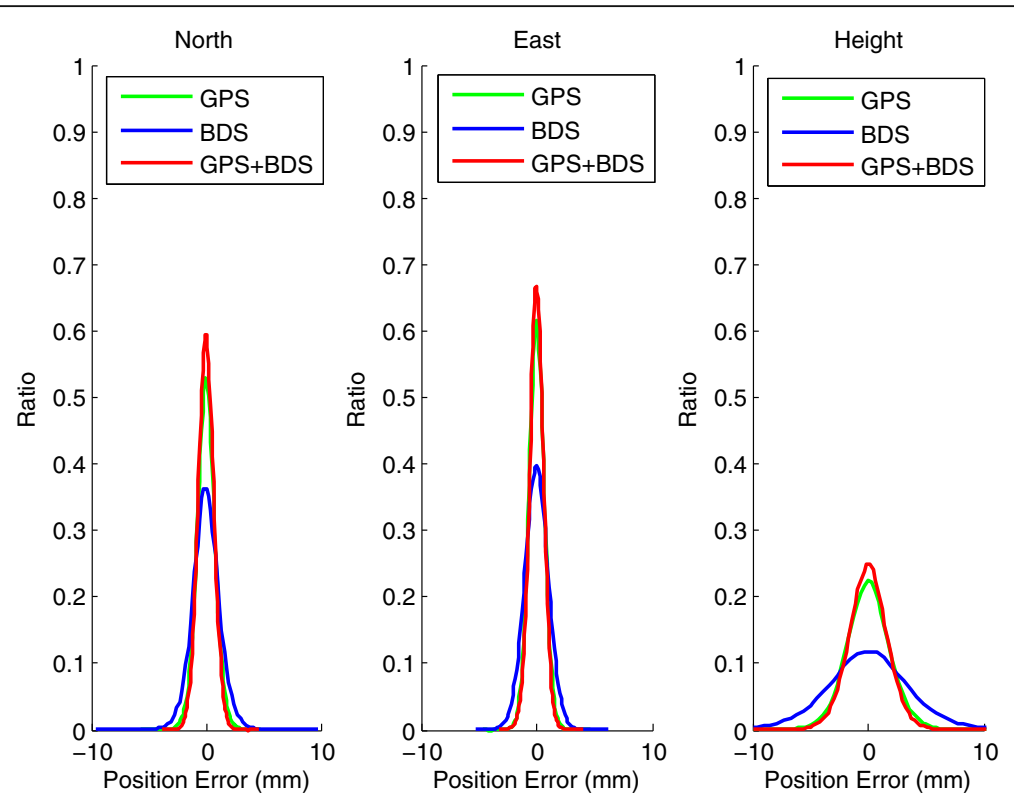

Fig. 10 Error distribution of the position time series (Figs. 5, 6, and 7) GPS-only, BDS-only and GPS + BDS

in that North-South and East-West position errors are much smaller than the height component.

Integration of GPS and BDS has a lot of advantages compared to positioning using GPS and BDS individually. Combining GPS and BDS could enhance the availability of satellites in some difficult situations where GPS-only or BDS-only would not work. As the precision of GPS relative positioning can be quite different from the one of $\mathrm{BDS}$, it is very important to analyze the precision of the combined use of GPS and BDS for precise positioning. Table 2 shows the RMS of the double difference relative positions for GPS-only, BDS-only and integration of GPS and BDS position errors in the three components for the whole-time series. The precision of integrating GPS and BDS position is better than GPS-only and BDS-only results. In some instances, both GPS-only and BDS-only have bigger errors. Figure 10 illustrates the distribution of the time series of positioning errors for GPS-only, BDSonly and the integration of GPS and BDS. This is the difference between the truth (zero baseline, and hence zero distance) and the actual results in the time series,

Table 2 GPS-only, BDS-only and GPS + BDS double difference position error Root Mean Squared value (RMS) in the different position components

\begin{tabular}{lccc}
\hline $\begin{array}{l}\text { components } \\
\text { Model }\end{array}$ & $\begin{array}{l}\text { North } \\
(\mathrm{mm})\end{array}$ & $\begin{array}{l}\text { East } \\
(\mathrm{mm})\end{array}$ & $\begin{array}{l}\text { Height } \\
(\mathrm{mm})\end{array}$ \\
\hline BDS-only & 1.22 & 1.03 & 3.70 \\
GPS-only & 0.81 & 0.67 & 1.94 \\
GPS + BDS & 0.69 & 0.62 & 1.71 \\
\hline
\end{tabular}

Figs. 5, 6, and 7. Zero baseline positioning results demonstrate that GPS-only position precision is currently better than BDS-only. As there are more available satellites being tracked at each epoch, more redundant observations during the least square adjustment could be expected to result in more accurate and reliable positions. It is shown that through integrating GPS and BDS it is possible to improve the precision of the two separate results.

\section{Conclusion}

In this paper, zero baseline observations were gathered in order to assess the accuracy of GPS-only, BDS-only and the integration of GPS and BDS relative positioning. There are on average $8 \mathrm{GPS}$ and 8 BDS satellites that could be tracked during the whole BDS orbital period at the University of Nottingham Ningbo, China. Sometimes the number of GPS satellites being tracked above an elevation cut-off angle of $15^{\circ}$ can drop down to only four during the experiments. The minimum number of BDS tracked during the experiments were six using the same elevation mask of $15^{\circ}$. The results demonstrate that BDS relative positioning accuracy could achieve millimetre level, but is still weaker than that of GPS. The integration of GPS and BDS could improve not only BDS-only, but also GPS-only position precisions.

From the derivation of observation equation, the positional error is caused by the precision of the observation, as well as the coefficient of observation equation. We will study this aspect by simulating more MEO satellites with the help of a SPIRENT GNSS simulator in the future. 


\section{Acknowledgements}

The work in this paper is supported by Young Scientist programme of Natural Science Foundation of China (NSFC) with a project code 41704024, as well as Ningbo Science and Technology Bureau - China as Part of the Project: Structural Health Monitoring of Infrastructure in the Logistics Cycle (2014A35008).

Availability of data and materials

Please contact the authors for data requests.

\section{Authors' contributions}

GWR obtained the Ningbo Science and Technology bureau funding that supported the research, and XT obtained the NSFC funding. XT carried out the field work and developed the algorithms. GWR and XT interpreted the results and co-wrote the paper. All authors read and approved the final manuscript.

\section{Authors' information}

Dr. Gethin Wyn Roberts is an Associate Professor in Geospatial Engineering at the University of the Faroe Islands. He was previously at the University of Nottingham as a staff member for 24 years, both in the UK and on the Ningbo, China campus.

Dr. Xu Tang is a research fellow at the University of Nottingham Ningbo, China. Prof Xiufeng He is a professor at HoHai University in Nanjing China.

\section{Competing interests}

The authors declare that they have no competing interests.

\section{Publisher's Note}

Springer Nature remains neutral with regard to jurisdictional claims in published maps and institutional affiliations.

\section{Author details}

${ }^{1}$ Faculty of Natural Sciences and Technology, University of the Faroe Islands, Tórshavn, Faroe Islands. ${ }^{2}$ The University of Nottingham Ningbo China,

Ningbo, China. ${ }^{3}$ HoHai University, Nanjing, China.

Received: 22 December 2017 Accepted: 13 April 2018

Published online: 25 April 2018

\section{References}

BeiDou-SIS-ICD-Test (2011) BeiDou navigation satellite system signal in space interface control document (Test Version), China Satellite Navigation Office

Deng C, Tang W, Liu J et al (2013) Reliable single-epoch ambiguity resolution for short baselines using combined GPS/BeiDou system. GPS Solutions 18(3): $375-386$

Fu X, Wu M (2011) Optimal design of broadcast ephemeris parameters for a navigation satellite system. GPS Solutions 16(4):439-448

Gao GX, Chen A, Lo S et al (2009) Compass-M1 broadcast codes in E2, E5b, and E6 frequency bands. leee J-Stsp 3(4):599-612

Grelier T, Dantepal J, Delatour A et al (2007) Initial observations and analysis of compass MEO satellite signals. Inside GNSS 2:39-43

Hauschild A, Montenbruck O, Sleewaegen JM et al (2012) Characterization of compass M-1 signals. GPS Solutions 16(1):117-126

He HB, Li JL, Yang YX et al (2014) Performance assessment of single- and dualfrequency BeiDou/GPS single-epoch kinematic positioning. GPS Solutions 18(3):393-403

Liu Z (2010) A new automated cycle slip detection and repair method for a single dual-frequency GPS receiver. J Geod 85(3):171-183

Odolinski R, Teunissen PJG, Odijk D (2014) Combined BDS, Galileo, QZSS and GPS single-frequency RTK. GPS Solutions 19(1):151-163

Shi C, Zhao Q, Hu Z, Liu J (2012) Precise relative positioning using real tracking data from compass geo and igso satellites. GPS Solutions 17(1):103-119

Tang X (2014) GPS/BDS precise positioning methods and applications, p 141

Tang X, He XF, Andam-Akorful SA (2014) The performance of Bds relative positioning usage with real observation data. Boletim De Ciencias Geodesicas 20(2):223-236

Teunissen PJG, Odolinski R, Odijk D (2014) Instantaneous BeiDou plus GPS RTK positioning with high cut-off elevation angles[]]. J Geod 88(4):335-350

Wang G, de Jong K, Zhao Q et al (2014) Multipath analysis of code measurements for BeiDou geostationary satellites. GPS Solutions 19(1):129-139

\section{Submit your manuscript to a SpringerOpen ${ }^{\circ}$ journal and benefit from:}

- Convenient online submission

Rigorous peer review

- Open access: articles freely available online

- High visibility within the field

- Retaining the copyright to your article

Submit your next manuscript at $\boldsymbol{\nabla}$ springeropen.com 